\title{
Prix de transfert optimaux et comportement stratégique des multinationales
}

\author{
Thierry Madiès* \\ Faculté des sciences économiques et sociales, \\ Université de Fribourg (Suisse)
}

\section{Introduction}

Le rôle des entreprises multinationales dans le commerce mondial a connu ces vingt dernières années un développement spectaculaire de sorte que plus de la moitié du commerce mondial se fait désormais entre sociétés apparentées. Comme l'a rappelé récemment l'OCDE (1995, pp. 3-4), dans ce contexte, "l'une des questions les plus difficile qui se soit posée concerne la fixation des prix de transferts entre sociétés apparentées ». Les prix de transfert sont les prix auxquels une entreprise transfère des biens corporels, ou rend des services à des entreprises associées. Si les prix de transfert sont importants aussi bien pour les contribuables que pour les administrations fiscales, c'est parce qu'ils déterminent dans une large mesure la répartition des bénéfices imposables entre entreprises apparentées relevant d'autorités fiscales différentes. ${ }^{1}$ Les gouvernements souscrivent généralement - souvent dans des traités bilatéraux - à la norme de pleine concurrence pour la fixa-

* Je tiens à remercier les deux rapporteurs anonymes qui m'ont permis d'améliorer une version antérieure de cet article ainsi que les participants à divers séminaires où cet article a été présenté. Adresse: Université de Fribourg, Faculté des Sciences économiques et sociales, avenue de l'Europe 20. Fribourg $\mathrm{CH}-1700$ tel : $0041(0) 263008220 \mathrm{Fax}$ : 0041(0)26 3009757 e-mail : thierry.madies@ unifr.ch

L'essentiel de cet article a été écrit quand l'auteur était chercheur associé au GRID (CNAS et Ecole normale supérieure de Cachan).

1 Alin d'empêcher certaines pratiques consistant à localiser à l'étranger des bénéfices normalement imposables en France, l'article 57 du CGI permet à l'administration de redresser les résultats déclarés par les entreprises françaises qui sont sous la dépendance ou, à linverse, qui ont le contrôle d'entreprises situées hors de France. En vertu de cette disposition, les bénéfices indirectement transférés à de telles entreprises établies hors de France, soit par voie de majoration des prix d'achat ou de diminution des prix de vente, soit par tout autre moyen, sont rapportés au résultat de l'entreprise résidente de France. Ce dispositif est également applicable aux entreprises placée sous la dépendance commune d'une méme entreprise, d'un 
tion des prix de transfert, ce qui signifie que les entreprises apparentées devraient traiter entre elles comme si elles n'avaient aucun lien de dépendance. Même si les pays s'entendent en général pour reconnaître la norme de pleine concurrence, certains ont des façons différentes de l'interpréter et d'appliquer les méthodes généralement acceptées. ${ }^{2}$

Une étude réalisée par Ernst \& Young (1995) sur un échantillon (non représentatif) de vingt-cinq entreprises multinationales ayant des activités dans huit pays différents (dont le Canada, les États-Unis, le Royaume-Uni et le Japon) fait clairement apparaître (1) que la question des prix de transfert est primordiale pour les multinationales de tous les pays étudiés (selon $80 \%$ des multinationales qui ont participé à l'étude, la fixation des prix de transfert serait leur problème fiscal international le plus important à l'heure actuelle); (2) que plus les multinationales sont susceptibles d'être soumises à une vérification fiscale de leur méthode de fixation des prix de transfert (83\% des entreprises interrogées ont déclaré que leurs méthodes de fixation des prix de transfert avaient déjà fait l'objet d'une vérification fiscale) et plus elles tiennent compte des réactions des autorités fiscales lorsqu'elles établissent ou révisent leur méthode; (3) que la plupart des multinationales connaissent bien le principe des accords de fixation préalable de prix de tranfert et s'attendent à ce qu'on y ait de plus en plus recours dans l'avenir. ${ }^{3}$

La littérature théorique sur les prix de transfert trouve ses fondements dans les travaux pionniers de Hirshleifer (1956), Copithorne (1971) et Horst (1971) sur les entreprises verticalement intégrées. Chez Hirshleifer, quand les décisions de production sont déléguées aux filiales, le choix de prix de transfert adéquats permet d'obtenir des filiales qu'elles choisissent un même niveau de production que si les décisions de production étaient prises de façon centralisée. Il s'agit donc de déterminer les prix de transfert de telle façon que le profit global de l'entreprise soit le plus élevé possible. Hirshleifer montre plus précisément que sous l'hypothèse d'indépendance de la demande et des choix de production entre filiales d'une même entreprise multinationale les prix de transfert sont égaux aux coûts marginaux de production. Horst (voir aussi Kant (1990) pour un modèle plus général) montre que les entreprises multinationales peuvent aussi utiliser les prix de transfert entre filiales situées dans des pays différents pour réduire leur charge fiscale

groupe ou d'un consortium, en même temps qu'une ou plusieurs entreprises étrangères. En revanche, la condition de dépendance ou de contrôle n'est pas exigée si le transfert est réalisé au profit d'entreprises établies dans un pays à fiscalité privilégiée. La notion de régime fiscal privilégié est celle retenue par l'article 238A du CGI qui prévoit que les personnes domiciliées ou établies hors de France sont regardées comme soumises à un régime fiscal privilégié dans l'État ou le territoire considéré si elles n'y sont pas imposables ou si elles y sont assujetties à des impôts sur les bénéfices ou les revenus notablement moins élevés qu'en France.

2 Les normes recommandées par l'OCDE sont fondées sur des méthodes transactionnelles : prix comparable sur le marché (PCM); prix de revente (resale minus); prix de revient majoré (cost plus); méthodes transactionnelles de la marge nette et du partage des bénéfices.

3 Pour une revue de la littérature économétrique sur la mantpulation par les entreprises multinationales des prix de transfert afin de minorer leur charge fiscale, voir Hines (1996). 
globale. Dans ce cas, la firme multinationale cherche à déterminer les prix de transfert qui lui permettent d'obtenir le niveau de profit après impôt le plus élevé possible. Dans ces conditions, une filiale située dans un pays où le taux d'impôt est faible et qui exporte vers une filiale (du même groupe) située dans un pays où le taux d'impôt est élevé devrait choisir le prix de transfert le plus élevé possible de façon à ce que les profits soient déclarés dans le pays où la fiscalité est la plus faible. ${ }^{4}$

L'objet de cet article s'éloigne des préoccupations de la littérature traditionnelle sur les prix de transfert dans le sens où son objectif est de faire le lien entre la littérature sur les prix de transfert et la littérature sur la politique commerciale stratégique (PCS). Rappelons que le modèle standard de PCS (voir Brander et Spencer (1985), Eaton et Grossman (1986) ou encore Brander (1995) pour une revue de la littérature sur le sujet) montre qu'un gouvernement peut accroître le bien-être national en offrant une subvention à l'exportation à son entreprise nationale quand celle-ci est en situation de concurrence à la Cournot avec une entreprises étrangère sur un marché tiers. La subvention à l'exportation permet à l'entreprise nationale de se comporter en leader de Stackelberg et d'accroître les quantités qu'elle vend au détriment de l'entreprise étrangère (du moins si l'on suppose que les quantités sont des substituts stratégiques). ${ }^{5}$ Nous montrons plus précisément qu'une multinationale peut sous certaines conditions manipuler ses prix de transfert de façon à capter des parts de marchés au détriment d'une entreprise tiers. Le prix de transfert joue alors un rôle analogue à celui d'une subvention à l'exportation.

Cette question est ignorée par la littérature sur les prix de transfert car les modèles en question reposent sur deux hypothèses essentielles qui éliminent d'emblée la possibilité de comportements stratégiques : la première est que les décisions concernant les quantités produites ou la politique de prix sont prises au niveau de la tête de groupe (autrement dit de façon centralisée) de façon à maximiser le profit global après impôt de la multinationale. Autrement dit, comme l'avait déjà souligné Bond (1980), il est fait l'hypothèse que les prix de transfert n'ont pas d'effet sur les décisions prises à la marge par les filiales. Cela suppose implicitement dans ce type de modèles qu'il existe deux sortes de prix de transfert : des prix de transfert qui sont utilisés à des fins d'optimisation fiscale et d'autres (égaux aux coûts marginaux) qui permettent de calculer les quantités optimales produites dans chaque filiales. La seconde hypothèse est que la multinationale est en situation de monopole sur les marchés nationaux où sont installées ses filiales. Une exception notable est l'article de Schjelderup et Sorgard

4 La manipulation des prix de cession internes, comme technique d'optimisation fiscale et de transfert de bénéfices, conduit inévitablement à une situation de concurrence fiscale entre États (voir sur ce point Hugounenq, Le Cacheux et Madiès (1999)). Pourtant, la littérature théorique sur cette question reste relativement peu développée par rapport à l'importante littérature sur la concurrence fiscale dont l'hypothèse centrale est la mobitité physique des bases imposables (pour une revue de la littérature voir par exemple Janeba (1997) et Delage (1999)).

5 Rappelons que si les entreprises sur le marché tiers se font une concurrence à la Bertrand, le résultat est inversé et le gouvernement taxe les exportations à l'équilibre. 
(1997) qui s'intéresse au cas où une multinationale est constituée d'une filiale qui est en situation de monopole sur le marché national et qui exporte un bien vers sa filiale située à l'étranger, cette dernière étant en situation de duopole à la Cournot avec une firme étrangère. Le principal résultat obtenu par les deux auteurs est que la multinationale choisit à l'équilibre un prix de transfert qui est généralement inférieur au coût marginal de production de la filiale exportatrice afin de rendre celle-ci plus agressive sur le marché étranger.

Le modèle que nous proposons constitue une généralisation du modèle de Schjelderup et Sorgard dans le sens où nous faisons l'hypothèse que les deux filiales de la multinationale (l'une située dans le pays d'origine et exportatrice de biens, l'autre située dans un pays étranger et importatrice de biens qu'elle revend sur le marché étranger à l'image d'un distributeur) sont engagées dans une compétition à la Cournot ou à la Bertrand sur leur marché respectif avec interactions entre les deux marchés par le biais des coûts de production de la filiale exportatrice. La prise en compte de comportements stratégiques fait que les deux filiales vont intégrer le prix de transfert dans leur décisions de production (dans une compétition à la Cournot) ou de prix (dans une compétition à la Bertrand). Plus précisément, nous construisons un modèle (1) dans lequel les prix de transfert (définis de façon centralisée par la tête de groupe) sont utilisés pour transférer des bénéfices entre filiales de façon à maximiser le profit net global de la multinationale, (2) dans lequel les filiales (qui sont des centres de profit) choisissent de façon décentralisée les quantités ou les prix optimaux sur les marchés oligopolistiques où elles opèrent.

Dans ces conditions, nous montrons que le prix de transfert qui permet à la multinationale de maximiser ses bénéfices consolidés est fonction de trois effets : (1) un effet «transfert de bénéfice » qui dépend de la comparaison des taux d'impôt entre les deux pays (et éventuellement du droit de douane dans le pays importateur); (2) un " effet stratégique " qui provient du fait que dans le cas d'une concurrence à la Cournot sur le marché étranger, un prix de transfert inférieur au coût marginal de production de la filiale exportatrice permet à la filiale importatrice située à l'étranger d'augmenter ses ventes et ses profits (et partant de là ceux de l'ensemble du groupe) en obligeant ses rivales à diminuer leur production; (3) un « effet de rétroaction » qui atténue l'incitation de la multinationale à sous-évaluer son prix de transfert car si les coûts marginaux de production de la filiale exportatrice sont croissants, une augmentation des quantités vendues par la filiale étrangère réduit la production de la filiale exportatrice sur son propre marché (et par là même ses profits). Ce dernier effet n'apparaît pas dans le modèle de Schjelderup et Sorgard car ils n'existe pas d'interactions entre les décisions prises par la filiale exportatrice sur le marché national et celles prises par la filiale importatrice située à l'étranger. La prise en compte de ces interactions transitant dans notre modèle par les coûts de production permet pourtant de renverser la proposition principale des deux auteurs selon laquelle le prix de transfert optimal est toujours inférieur au coût marginal 
de production dans une configuration de concurrence à la Cournot.

L'article est organisé comme suit. La section 2 présente le modèle standard et discute des hypothèses sous-jacentes quand les deux filiales sont en situation de monopole sur leur marché respectif. La sections 3 détermine le prix de transfert optimal quand les deux filiales de la multinationale font face à une concurrence avec des entreprises identiques sur leur marché respectif et qu'elles choisissent les quantités produites de façon décentralisée. La section 4 s'intéresse au cas où la filiale exportatrice a des coûts de production différents de ceux des entreprises qui sont en concurrence avec sa filiale sur le marché étranger et aux conséquences d'une telle hypothèse sur la détermination du prix de transfert optimal. La section 5 traite de la question de la forclusion quand la multinationale fournit un bien de consommation intermédiaire à l'entreprise étrangère et que celle-ci est en situation de concurrence avec son distributeur étranger (autrement dit sa filiale étrangère). La section 6 détermine le prix de transfert optimal quand il y a concurrence en prix et non plus à la Cournot.

\section{Modèle de référence et résultats préliminaires}

Des auteurs comme Eden (1978) ou Lall (1978) ont avancé l'idée que l'existence même d'opérations entre entreprises affiliées répondait au souci des entreprises multinationales de réduire le montant global d'impôt versé aux différentes administrations publiques. Aussi l'objet de cette première section est de montrer de façon simple comment l'utilisation de prix de transfert (en l'occurrence ici des prix de cession internes) entre entreprises apparentées peut permettre à une entreprise multinationale de localiser une partie de ses bénéfices dans des pays où la fiscalité est la plus faible ("profit shifting").

Le modèle que nous présentons s'intéresse à une entreprise multinationale qui possède deux filiales et dont l'objectif est de maximiser son profit net mondial. La première filiale est implantée dans le pays d'origine (pays A) de la société-mère (cette dernière n'ayant pas d'autre rôle dans le modèle que de calculer les prix de transfert optimaux). La seconde filiale est installée dans un pays étranger (pays B) où elle revend le produit final qu'elle importe de la filiale situé dans le pays A. On suppose dans cette section que les deux filiales sont en situation de monopole sur leur marché respectif (hypothèse que l'on lèvera par la suite). La filiale située dans le pays A produit les quantités $g$ et $Q_{1}$ et elle a une fonction de coût $c\left(q+Q_{1}\right)$ avec $c^{\prime} \geqslant 0$ et $c^{\prime \prime} \geqslant 0$ (rendements d'échelle décroissants). La quantité $q$ est vendue directement dans le pays $\mathrm{A}$ au prix $p(q)$, ce qui lui rapporte des recettes $r(q) \equiv p(q) q$ avec $r^{\prime \prime} \leqslant 0$ et $p^{\prime}<0$. La quantité $Q_{1}$ est exportée vers la filiale située dans le pays $B$ avec un prix de transfert égal à $m$. La filiale revend ensuite le bien sur le marché étranger à un prix $P\left(Q_{1}\right)$, ce qui lui rapporte des recettes égales à $R\left(Q_{1}\right) \equiv P\left(Q_{1}\right) Q_{1}$ avec $P^{\prime}<0$ et $R^{\prime \prime} \leqslant 0$. On suppose enfin que la multinationale peut faire de la discrimination par 
les prix sur le marché d'origine et sur le marché étranger de sorte que les fonctions de profit des deux filiales peuvent s'écrire:

$$
\pi^{A}=\left[r(q)-c\left(q+Q_{1}\right)+m Q_{1}\right] \text { et } \pi^{B}=\left[R\left(Q_{1}\right)-m(1+\tau) Q_{1}\right]
$$

Les deux filiales sont soumises à un impôt sur le bénéfice prélevé à la source au taux $t$ pour le pays $\mathrm{A}$ et au taux $T$ pour le pays $\mathrm{B}$. Ce dernier est supposé imposer en outre un droit de douane ad valorem sur ses importations au taux $\tau$. On suppose enfin que les impôts payés par la filiale étrangère à l'administration fiscale du pays où elle est installée font l'objet d'un crédit d'impôt dans le pays de résidence au moment où ils sont rapatriés (ce qui est conforme au modèle de double imposition préconisé par l'OCDE).

Conformément à la littérature précédente, on suppose dans cette section que les décisions concernant le prix de transfert et les quantités produites sont prises au niveau de la société-mère (hypothèse $\mathrm{H} 1$ ) et que les deux filiales sont en situation de monopole sur le marché où elles opèrent (hypothèse H2). Deux cas de figure peuvent alors se présenter selon qu'il existe un crédit d'impôt partiel (hypothèse H3) ou complet (hypothèse H4). Cette distinction n'est pas anodine car, dans le premier cas, on est en présence d'un système de taxation selon le principe de la source alors que dans le second cas, le principe d'assujettissement est le principe de résidence (dit aussi principe du bénéfice mondial) avec crédit d'impôt complet pour les revenus de source étrangère. ${ }^{6}$

(A) H3:t<T - Dans ce cas, les revenus d'origine étrangère sont exonérés d'impôt car le crédit d'impôt est limité au montant d'impôt que supporteraient ces revenus s'ils étaient taxés au taux du pays de résidence. Les profits agrégés réalisés par la multinationale sont égaux à :

$$
\Pi^{G}=(1-t) \pi^{A}+(1-T) \pi^{B}
$$

En l'absence de tout comportement stratégique, le seul rôle du prix de transfert est de permettre un transfert de bénéfice dans le pays offrant les conditions fiscales et douanières les plus avantageuses. On suppose cependant que le prix de transfert est borné inférieurement par le fait que le profit de la filiale exportatrice ne peut pas être négatif et supérieurement par le fait que le profit de la filiale importatrice située à l'étranger ne peut pas non plus être négatif: $m \in\left[(r(q)-c) / Q_{1}, P /(1+\tau)\right]$. Le prix de transfert optimal dépend du signe de l'équation suivante:

$$
\frac{d \Pi^{G}}{d m}=Q_{1}[(1-t)-(1-T)(1+\tau)]=Q_{1}(1-T)(\phi-\tau)
$$

avec $\phi \equiv(T-t) /(1-T)$.

6 Pour une présentation des mesures visant à limiter la double imposition des revenus transfrontaliers, voir par exemple Hugounenq, Le Cacheux et Madiès (1999). 
Lemme 1 Sous les hypothèses (H1)-(H2)-(H3), le signe de $d \Pi^{C} / d m$ dépend de la comparaison de $\phi$ et $\tau$. Si $\phi>\tau$ alors les profits sont une fonction croissante du prix de transfert $\left(d \Pi^{G} / d m>0\right)$ et la multinationale réduit le montant global d'impôt qu'elle paie en choisissant un prix de transfert élevé (supérieur au coût marginal): dans ce cas le prix de transfert optimal correspond à la borne supérieure. $A$ l'inverse, quand $\phi<\tau, d \Pi^{G} / d m<0$ et la multinationale a intérêt à choisir un prix de transfert faible (inférieur au coût marginal) : le prix de transfert optimal est égal à la borne inférieure.

(B) $\mathrm{H} 4: t>T-$ Dans ce cas, les profits réalisés par la filiale sont taxés au taux d'impôt du pays de résidence de la société-mère, autrement dit dans le pays $A$ :

$$
\Pi^{G}=(1-t)\left[\pi^{A}+\pi^{B}\right]
$$

Le prix de transfert optimal choisi par la multinationale dépend du signe de l'équation suivante :

$$
\frac{d \Pi^{G}}{d m}=-\tau Q_{1}(1-t)<0
$$

Lemme 2 Sous les hypothèses (H1) $\left(\mathrm{H}_{2}\right) \Gamma\left(\mathrm{H}_{4}\right)$, la multinationale choisit toujours un prix de transfert le plus faible possible afin de réduire le paiement du droit de douane et la borne inférieure est atteinte.

\section{Concurrence a la Cournot et prix de transfert optimaux}

On suppose maintenant (1) qu'au moins une des deux filiales est en situation de concurrence à la Cournot sur son marché; (2) que la société-mère détermine de façon centralisée les prix de transfert optimaux de façon à maximiser les profits nets globaux de la multinationale; (3) que les filiales déterminent de façon décentralisée les quantités produites.

Plus précisément, la filiale située en A vend la quantité $q_{1}$ sur un marché oligopolistique où opèrent $(M-1)$ autres entreprises identiques ( $M$ est supposé exogène). Ses ventes rapportent à la filiale du pays $\mathrm{A}$ des recettes égales à $r_{1}\left(q_{1}, q_{-1}\right)=p\left(q_{1}+q_{-1}\right) q_{1} \cdot q_{-1}$ représente les quantités vendues par les $(m-1)$ autres entreprises $\left(q_{-1}=\sum_{j \neq 1} q_{j}\right)$. On suppose en outre que $\partial^{2} r_{1} / \partial^{2} q_{1} \leqslant 0$ et $\partial r_{1} / \partial q_{j}<0 \forall j \neq 1$. Cette dernière hypothèse implique que les produits vendus dans le pays A sont des substituts stratégiques. De la même façon, la filiale dans le pays $\mathrm{B}$ vend la quantité $Q_{1}$ sur un marché oligopolistique où opèrent $(N-1)$ autres entreprises identiques. Ses ventes rapportent à la filiale du pays $\mathrm{B}$ des recettes égales à $R_{1}\left(Q_{1}, Q_{-1}\right) \equiv$ $P\left(Q_{1}+Q_{-1}\right) Q_{1} \cdot Q_{-1}$ représente les quantités vendues par les $(N-1)$ autres 
entreprises $\left(Q_{-1}=\sum_{l \neq 1} Q_{l}\right)$. On suppose en outre que $\partial^{2} R_{1} / \partial^{2} Q_{1} \leqslant 0$ et $\partial R_{1} / \partial Q_{l}<0 \forall l \neq 1$.

A partir du moment où les décisions concernant le prix de transfert sont prises de façon centralisée mais que les décisions de production sont déléguées aux filiales, on est en présence d'un jeu à deux étapes. À la première étape, la société-mère choisit $m$ et à la seconde étape les $(M+N)$ entreprises, qui sont supposées observer parfaitement $m$ choisissent les quantités produites sur leur marché. La résolution du jeu se fait selon la méthode de la récurrence amont : on commence par déterminer les quantités d'équilibre comme des fonctions de $m$ puis on se sert de ces dernières pour calculer le prix de transfert optimal à la première étape. Comme précédemment, il convient de distinguer le cas où il y a un crédit d'impôt partiel du cas où il y a un crédit d'impôt complet.

(A) - H3 : prix de transfert optimal quand $t<T$

Rappelons que le profit net global de la multinationale est dans ce cas égal à :

$$
\begin{aligned}
\Pi^{G}= & (1-t) \pi_{1}^{A}+(1-T) \pi_{1}^{B} \\
= & (1-t)\left[r_{1}\left(q_{1}, q_{-1}\right)-c\left(q_{1}+Q_{1}\right)+m Q_{1}\right] \\
& +(1-T)\left[R_{1}\left(Q_{1}, Q_{-1}\right)-m(1+\tau) Q_{1}\right]
\end{aligned}
$$

L'ensemble des entreprises observant parfaitement le prix de transfert fixé par la multinationale et le considérant comme exogène, les conditions du premier ordre les concernant sont :

- Sur le marché national:

$$
\begin{aligned}
& \frac{\partial \pi_{1}^{A}}{\partial q_{1}}=(1-t)\left[\frac{\partial r_{1}}{\partial q_{1}}-\frac{\partial c}{\partial q_{1}}\right]=0 \\
& \frac{\partial \pi_{j}^{A}}{\partial q_{j}}=(1-t)\left[\frac{\partial r_{j}}{\partial q_{j}}-\frac{\partial c}{\partial q_{j}}\right]=0 \quad \forall j \neq 1
\end{aligned}
$$

On suppose que les conditions du second ordre sont vérifiées, ce qui garantit l'existence de l'équilibre de Nash: $\partial^{2} \pi_{1}^{A} / \partial q_{1} \partial q_{1}<0$ et $\partial^{2} \pi_{j}^{A} / \partial q_{j} \partial q_{j}<0$ $\forall j \neq 1$. On sait en outre que dans le cas d'un bien homogène, les relations suivantes sont vérifiées: $\quad \partial^{2} \pi_{i}^{A} / \partial q_{1} \partial q_{1}<\partial^{2} \pi_{1}^{A} / \partial q_{1} \partial q_{j}<0 \quad$ et $\partial^{2} \pi_{j}^{A} / \partial q_{j} \partial q_{j}<\partial^{2} \pi_{j}^{A} / \partial q_{j} \partial q_{1}<0 \forall j \neq 1$. Ces conditions sont suffisantes pour s'assurer que l'équilibre de Nash est unique et stable sur le marché national.

- Sur le marché étranger :

$$
\begin{aligned}
& \frac{\partial \pi_{1}^{B}}{\partial Q_{1}}=(1-T)\left[\frac{\partial R_{1}}{\partial Q_{1}}-(1+\tau) m\right]=0 \\
& \frac{\partial \pi_{l}^{B}}{\partial Q_{l}}=(1-T)\left[\frac{\partial R_{l}}{\partial Q_{l}}-\frac{\partial C}{\partial Q_{l}}\right]=0 \quad \forall l \neq 1
\end{aligned}
$$


Les conditions du second ordre vérifient : $\quad \partial^{2} \pi_{1}^{B} / \partial Q_{1} \partial Q_{1}<0 \quad$ et $\partial^{2} \pi_{l}^{B} / \partial q_{l} \partial q_{l}<0 \quad \forall l \neq 1 ; \quad \partial^{2} \pi_{1}^{B} / \partial Q_{1} \partial Q_{1}<\partial^{2} \pi_{1}^{B} / \partial Q_{1} \partial Q_{l}<0 \quad$ et $\partial^{2} \pi_{l}^{B} / \partial Q_{l} \partial Q_{l}<\partial^{2} \pi_{l}^{B} / \partial Q_{l} \partial Q_{1}<0 \forall l \neq 1$.

Les conditions précédentes nous permettent de calculer implicitement les quantités d'équilibre sur chacun des deux marchés comme des fonctions de $m$. En différentiant les conditions (6a) et $(6 \mathrm{~b})$ et en supposant que toutes les firmes sont identiques, on obtient:

$\frac{\partial q_{1}}{\partial m}=-\frac{c^{\prime \prime}}{D} \cdot \frac{\partial Q_{1}}{\partial m} \cdot \frac{\partial^{2} \pi_{1}^{A}}{\partial q_{j} \partial q_{j}}<0 ; \quad \frac{\partial q_{j}}{\partial m}=\frac{c^{\prime \prime}}{D} \cdot \frac{\partial^{2} \pi_{j}^{A}}{\partial q_{j} \partial q_{1}} \cdot \frac{\partial Q_{1}}{\partial m}>0 \quad \forall j \neq 1$

où $D=\frac{\partial^{2} \pi_{1}^{A}}{\partial q_{1} \partial q_{1}} \cdot \frac{\partial^{2} \pi_{j}^{A}}{\partial q_{j} \partial q_{j}}-\frac{\partial^{2} \pi_{i}^{4}}{\partial q_{1} \partial q_{j}} \cdot \frac{\partial^{2} \pi_{j}^{A}}{\partial q_{j} \partial q_{1}}>0$ par hypothèse.

(8a) montre qu'une baisse du prix de transfert diminue les quantités vendues par la filiale exportatrice mais augmente les quantités vendues par les entreprises qui lui font concurrence sur le marché domestique.

On obtient de la même façon en différentiant (7a) et (7b) :

$\frac{\partial Q_{1}}{\partial m}=\frac{(1+\tau)}{D^{*}} \cdot \frac{\partial^{2} \pi_{l}^{B}}{\partial Q_{l} \partial Q_{l}}<0 ; \quad \frac{\partial Q_{l}}{\partial m}=-\frac{(1+\tau)}{D^{*}} \cdot \frac{\partial^{2} \pi_{l}^{B}}{\partial Q_{l} \partial Q_{1}}>0 \quad \forall l \neq 1$ où $D^{*}=\frac{\partial^{2} \pi_{1}^{B}}{\partial Q_{1} Q_{1}} \cdot \frac{\partial^{2} \pi_{j}^{B}}{\partial Q_{l} \partial Q_{l}}-\frac{\partial^{2} \pi_{1}^{B}}{\partial Q_{1} Q q_{l}} \cdot \frac{\partial^{2} \pi_{l}^{B}}{\partial Q_{l} \partial Q_{1}}>0$ par hypothèse.

(8b) montre qu'une baisse du prix de transfert augmente les quantités vendues par la filiale importatrice et diminue les quantités vendues par les firmes qui lui font concurrence sur le marché étranger.

L'effet d'un changement à la marge de $m$ sur le profit net global est alors déterminé comme suit:

$$
\begin{aligned}
\frac{d \Pi^{G}}{d m} & =(1-t)\left[\left(\frac{\partial r_{1}}{\partial q 1}-\frac{\partial c}{\partial q_{1}}\right) \frac{\partial q_{1}}{\partial Q_{1}} \cdot \frac{\partial Q_{1}}{\partial m}+\sum_{j \neq 1}^{M} \frac{\partial r_{1}}{\partial q_{j}} \cdot \frac{\partial q_{j}}{\partial q_{1}} \cdot \frac{\partial q_{1}}{\partial Q_{1}} \cdot \frac{\partial Q_{1}}{\partial m}\right. \\
& \left.-\left(\frac{\partial c}{\partial Q_{1}} \cdot \frac{\partial Q_{1}}{\partial m}\right)+Q_{1}+m \cdot \frac{\partial Q_{1}}{\partial m}\right]+(1-T)\left[\left(\frac{\partial R_{1}}{\partial Q_{1}}-m \cdot(1+\tau)\right) \frac{\partial Q_{1}}{\partial m}\right. \\
& \left.+\sum_{l \neq 1}^{N} \frac{\partial R_{1}}{\partial Q_{l}} \cdot \frac{\partial Q_{l}}{\partial Q_{1}} \cdot \frac{\partial Q_{1}}{\partial m}-(1+\tau) Q_{1}\right]
\end{aligned}
$$

Si l'on fait l'hypothèse que les entreprises dans le pays $A$ (respectivement dans le pays B) sont toutes identiques et que l'on remplace (6a) et (7a) dans (9), on obtient l'expression suivante après avoir réarrangé les termes dans l'expression (9) de façon à faire apparaître le markup : 


$$
\begin{gathered}
m-\frac{\partial c}{\partial Q_{1}}=\frac{1-T}{1-t}\left[\frac{(\tau-\phi) Q_{1}}{\partial Q_{1} / \partial m}-(M-1)\left(\frac{1-t}{1-T}\right) \cdot \frac{\partial r_{1}}{\partial q_{j}} \cdot \frac{\partial q_{j}}{\partial q_{1}} \cdot \frac{d q_{1}}{d Q_{1}}\right. \\
\left.-(N-1) \frac{\partial R_{1}}{\partial Q_{l}} \cdot \frac{\partial Q_{l}}{\partial Q_{1}}\right]
\end{gathered}
$$

On obtient immédiatement la proposition 1 :

Proposition 1 Quand les entreprises se livrent à une concurrence à la Cournot et que les deux filiales déterminent leurs décisions de production de façon décentralisée, le signe de $\left(m-\partial c / \partial Q_{1}\right)$ dépend de trois effets qui jouent en sens opposé :

(i) un effet "transfert de bénéfice ": $\left[\frac{(\tau-\phi) Q_{1}}{\partial Q_{1} / \partial m}\right]<0(>0)$ si $\tau>\phi(\tau<\phi)$.

(ii) un effet "stratégique» sur le marché étranger:

$$
-\left[(N-1) \frac{\partial R_{1}}{\partial Q_{l}} \cdot \frac{\partial Q_{l}}{\partial Q_{1}}\right]<0
$$

(iii) un effet rétroactif sur le marché national:

$$
-\left[(M-1)\left(\frac{1-t}{1-T}\right) \cdot \frac{\partial r_{1}}{\partial q_{j}} \cdot \frac{\partial q_{j}}{\partial q_{1}} \cdot \frac{d q_{1}}{d Q_{1}}\right]>0
$$

Preuve - Le signe de (i) est négatif si $\tau>\phi$ et positif si $(\tau<\phi)$ car $\partial Q_{1} / \partial m<0$ d'après (8b). (ii) est négatif car (1) dans l'hypothèse d'une concurrence à la Cournot, il est bien connu qu'il existe un grand nombre de fonctions de demande telles que $\partial Q_{1} / \partial Q_{1}<0$ (une condition suffisante pour que les autres entreprises diminuent leurs ventes quand la filiale située à l'étranger augmente ses ventes est que les fonctions de coût et de demande soient convexes) et (2) parce que, par hypothèse, les quantités produites dans le pays $\mathrm{B}$ par les $\mathrm{N}$ entreprises sont des substituts stratégiques $\left(\partial R_{1} / \partial Q_{l}<\right.$ $0)$. (iii) est positif car, (1) $\partial q_{j} / \partial q_{1}<0$ par hypothèse, (2) $\partial r_{1} / \partial q_{j}<0$ et (3) car $d q_{1} / d Q_{1}<0$. Le signe de $d q_{1} / d Q_{1}$ est obtenu en différenciant (7a) par rapport à $q_{1}$ et $Q_{1}: \frac{d q_{1}}{d Q_{1}}=\frac{c^{\prime \prime}}{\partial^{2} \pi_{1}^{4} / \partial q_{1} \partial q_{1}}<0$.

Corrolaire 1 Si les coûts marginaux de production de la filiale exportatrice sont constants, alors l'effet " coût " disparaît $\left(d q_{1} / d Q_{1}=0\right)$. Les seuls effets qui subsistent sont l'effet " transfert de bénéfice » et l'effet "stratégique ".

Les effets mis en exergue dans la proposition 1 peuvent être expliqués de la façon suivante:

- L'effet « transfert de bénéfice " ( profit shifting effect ») incite la multinationale à choisir un prix de transfert faible (c'est-à-dire inférieur au coût marginal de production de la filiale exportatrice: $\left.m<\partial c / \partial Q_{1}\right)$ quand $\phi<\tau$ et un prix de transfert plus élevé quand $\phi>\tau\left(m>\partial c / \partial Q_{1}\right)$. Cet effet est le seul qui subsiste quand les deux filiales sont en situation 
de monopole sur leur marché respectif $(M=N=1)$. Dans le cas où $t=T=0$, le prix de transfert est égal au coût marginal de production de la filiale exportatrice (on retrouve en cela le résultat bien connu de Hirsheifer (1956)).

Cependant, dès lors que l'on autorise une concurrence à la Cournot sur l'un ou l'autre des marchés, alors le résultat standard de Hirsheifer ne tient plus, même en l'absence d'imposition et de droits de douane à cause des effets stratégique et de rétroaction :

- L'effet «stratégique », de signe négatif, peut être interprété de la façon suivante : un prix de transfert plus faible que le coût marginal (que l'on suppose parfaitement observable par l'ensemble des entreprises présentes sur le marché) permet à la filiale installée dans le pays $\mathrm{B}$ de vendre une quantité plus élevée de produits. Les entreprises concurrentes anticipent cela et leur meilleure réponse consiste à réduire leur ventes (hypothèse de substituabilité stratégique) de sorte que le profit de la filiale installée dans le pays $\mathrm{B}$ augmente (ainsi que le profit global de la multinationale).

Proposition 2 Quand $\tau \geqslant \phi(y$ compris $t=T=0)$ et quand la filiale exportatrice est en situation de monopole $(M=1)$ ou que ses coûts marginaux sont constants, alors il est toujours vrai que $m<\partial c / \partial Q_{1}$.

- L'effet rétroactif repose sur l'hypothèse de rendements d'échelle décroissants et peut s'interpréter de la façon suivante : une augmentation des quantités vendues par la filiale dans le pays étranger $\left(Q_{1}\right)$ accroît le coût marginal de production de la filiale exportatrice située dans le pays $A$ et conduit celle-ci à réduire ses quantités vendues. Dans ces conditions, les autres entreprises en profitent pour augmenter leurs ventes («effet «stratégique »). Toutes choses égales par ailleurs, les profits de la filiale du pays $A$ baissent (ce qui fait baisser les profits globaux de la multinationale). L'effet de rétroactif et l'effet stratégique ont par conséquent un impact opposé sur le prix de transfert optimal. ${ }^{7}$

(B) $\mathrm{H} 4: t>T$

Dans ce cas, la fonction de profit global de la multinationale est donnée par :

$$
\begin{aligned}
\Pi^{G}= & (1-t)\left[\pi_{1}^{A}+\pi_{1}^{B}\right] \\
= & (1-t)\left[\left(r_{1}\left(q_{1}, q_{-1}\right)-c_{1}\left(q_{1}+Q_{1}\right)+m Q_{1}\right)\right. \\
& \left.+\left(R_{1}\left(Q_{1}, Q_{-1}\right)-m(1+\tau) Q_{1}\right)\right]
\end{aligned}
$$

\footnotetext{
7 Remarquons que le signe des deux derniers effets dépend des hypothèses généralement admises de substituabilité stratégique des quantilés produites et de rendements décroissants à l'échelle. Cependant, si les quantités étaient des compléments stratégiques, le signe de l'eflet stratégique et de l'effet rétroactif sur le marché national seraient inversés dans la proposition 1 (sauf à supposer que les biens sont des compléments ordinaires sur les deux marchés, auquel cas la proposition 1 reste vatide). De la méme façon, sous l'hypothèse de rendements d'échelle croissants (et non plus décroissants), l'eftet rétroactif viendrait dans tous les cas renforcer l'elfet stratégique dans le sens d'un prix de transfert sous-évalué (c'est-à-dire inférieur au coût marginal).
} 
On obtient de la même façon le prix de transfert optimal :

$$
\begin{gathered}
m-\frac{\partial c}{\partial Q_{1}}=\left[\frac{\tau Q_{1}}{\partial Q_{1} / \partial m}-(M-1) \cdot \frac{\partial r_{1}}{\partial q_{j}} \cdot \frac{\partial q_{j}}{\partial q_{1}} \cdot \frac{d q_{1}}{d Q_{1}}\right. \\
\left.-(N-1) \frac{\partial R_{1}}{\partial Q_{l}} \cdot \frac{\partial Q_{l}}{\partial Q_{1}}\right]
\end{gathered}
$$

Lemme 3 Quand les impôts payés par la filiale importatrice au pays étranger font l'objet d'un crédit d'impôt complet, alors l'effet " transfert de bénéfice " et l'effet stratégique vont tous les deux dans le sens d'un prix de transfert minoré, c'est-à-dire inférieur au coût marginal de production de la filiale exportatrice.

Le lemme 3 permet d'obtenir directement la proposition 3 :

Proposition 3 Si la filiale exportatrice est en situation de monopole ( $M=$ 1) sur le marché national ou si son coût marginal de production est non croissant, alors il est toujours vrai que $m<\partial c / \partial Q_{1}$ quand $t>T$.

\section{Prix de transfert optimaux et coûts asymétriques : le cas de fonctions de demande linéaire}

Nous avons supposé jusqu'à présent que les entreprises étaient toutes identiques. La question que nous nous posons maintenant est de savoir comment la multinationale choisit son prix de transfert optimal quand les entreprises concurrentes de sa filiale étrangère ont un coût marginal de production $\left(c^{*}\right)$ différent de celui de la filiale exportatrice $(c)$. Pour en donner une intuition, nous supposerons que le coût marginal de production de la filiale exportatrice, $c$, est constant (ce qui permet d'éliminer l'effet rétroactif sur le marché national transitant par les coûts de production). On supposera par ailleurs que la demande est linéaire (de la forme $P=a-\left(Q_{1}+Q_{2}\right)$ ) et que la filiale importatrice est en situation de duopole sur le marché étranger $(N=2))$.

Dans le cas précis, les conditions du premier ordre données par les équations (6a) et (6.b) permettent de calculer de façon simple la quantité d'équilibre $Q_{1}$ (mais aussi $Q_{2}$ ) comme une fonction des paramètres de coûts et de demande ainsi que du prix de transfert :

$$
Q_{1}=\frac{-2 m+c^{*}+a}{3}
$$

Par ailleurs, l'équation (9) s'écrit sous ce jeu d'hypothèses :

$$
\begin{aligned}
m= & {\left[c-\frac{c^{*}+a}{2} \cdot\left(\left(\frac{\tau-\phi}{1+\tau}\right)+\frac{1}{3}\right)\right] } \\
& \cdot\left[1-\left(\frac{1-T}{1-t}\right)\left(\left(\frac{\tau-\phi}{1+\tau}\right)+\frac{1}{3}\right)\right]^{-1}
\end{aligned}
$$


Si l'on suppose que $m$ est positif et que $t=T=\tau=0$ de façon à éliminer tout transfert de bénéfice résultant de considérations fiscales, on trouve immédiatement que :

$$
m=\frac{3}{2} c-\frac{1}{4} c^{*}-\frac{1}{4} a
$$

L'équation précédente montre que le prix de transfert optimal est une fonction croissante du coût marginal de production de la filiale exportatrice et une fonction décroissante du coût marginal de production de l'entreprise qui est en concurrence avec la filiale située à l'étranger.

Proposition 4 Dans un modèle de duopole à la Cournot caractérisé par (i) une fonction de demande linéaire, (ii) des coûts marginaux constants et (iii) des taux d'impôt et un droit de douane nuls, la multinationale fixe un prix de transfert d'autant plus bas que le coût marginal de la filiale exportatrice est faible par rapport à celui de l'entreprise étrangère.

La proposition 4 fait apparaître un résultat que l'on peut rapprocher de celui obtenu par Neary (1994) dans le cadre d'un modèle de politique commerciale stratégique. Le prix de transfert, à l'image d'une subvention à l'exportation, permet à l'entreprise multinationale d'augmenter les profits de l'entreprise nationale au détriment de l'entreprise étrangère. Dans ces conditions, plus le coût marginal de production de la filiale exportatrice est faible et plus la filiale située à l'étranger a un avantage important en terme de coût par rapport à sa concurrente. Ce résultat apparemment paradoxal ne l'est pas : l'efficacité de la politique de profit shifting de la multinationale (au détriment de l'entreprise concurrente de sa filiale étrangère) sera d'autant plus importante que la filiale étrangère est compétitive par rapport à sa concurrente.

\section{$5 \quad$ Forclusion et prix de transfert optimaux}

Le prix de transfert était utilisé jusqu'à présent par la multinationale dans le seul but de rendre plus compétitive sa filiale située à l'étranger par rapport à ses rivales. Nous supposons dans la suite que la multinationale exporte vers sa filiale située à l'étranger un bien final mais qu'elle approvisionne en outre l'entreprise étrangère en input intermédiaire que celle-ci transforme pour produire le bien final. Se pose dès lors la question de la forclusion car la multinationale peut utiliser son pouvoir de monopole sur l'input intermédiaire et se livrer à une «extorsion par les prix » de façon à évincer l'entreprise étrangère du marché du bien final.

Si l'on note $m^{*}$ le prix auquel la multinationale vend l'input intermédiaire à l'entreprise étrangère, alors le profit net de cette dernière s'écrit :

$$
\pi_{2}^{B}=(1-T)\left[R_{2}\left(Q_{1}+Q_{2}\right)-m^{*}(1+\tau) Q_{2}\right]
$$


La filiale exportatrice étant supposée vendre la totalité de sa production à sa filiale située à l'étranger et à l'entreprise concurrente de celle-ci, sa fonction de profit devient:

$$
\pi_{1}^{A}=(1-t)\left[m Q_{1}+m^{*} Q_{2}-c\left(Q_{1}+Q_{2}\right)\right]
$$

La fonction de profit de la filiale importatrice n'ayant pas changé par rapport à précédemment, le profit net global de la multinationale s'écrit de la façon suivante :

$$
\Pi^{G}=(1-t) \pi_{1}^{A}+(1-T) \pi_{1}^{B}
$$

Le jeu se résout encore par récurrence amont: la filiale située à l'étranger et l'entreprise concurrente choisissent les quantités produites de façon à maximiser leur profit. Les conditions du premier ordre sont les suivantes:

$$
\begin{aligned}
& \frac{\partial \pi_{1}^{B}}{\partial Q_{1}}=(1-T)\left[\frac{\partial R_{1}}{\partial Q_{1}}-(1+\tau) m\right]=0 \\
& \frac{\partial \pi_{2}^{B}}{\partial Q_{2}}=(1-T)\left[\frac{\partial R_{2}}{\partial Q_{2}}-(1+\tau) m^{*}\right]=0
\end{aligned}
$$

Les conditions du premier ordre permettent de déterminer les quantités d'équilibre comme des fonctions du prix de transfert et du prix de l'input intermédiaire : $Q_{1}\left(m, m^{*}\right)$ et $Q_{2}\left(m, m^{*}\right)$. On peut montrer facilement en différentiant les conditions $(15 \mathrm{a})$ et $(15 \mathrm{~b})$ que :

$$
\frac{\partial Q_{1}}{\partial m}<0, \quad \frac{\partial Q_{1}}{\partial m^{*}}>0, \quad \frac{\partial Q_{2}}{\partial m}>0, \quad \frac{\partial Q_{2}}{\partial m^{*}}<0
$$

Autrement dit, une augmentation du prix de transfert réduit la production de la filiale située à l'étranger mais augmente les quantités produites par l'entreprise concurrente. $\grave{A}$ l'inverse, une augmentation du prix de l'input intermédiaire augmente les quantités produites par la filiale importatrice et diminue celles de l'entreprise concurrente.

À la première étape, la multinationale détermine à la fois le prix de transfert $(m)$ et le prix de l'input intermédiaire $\left(m^{*}\right)$ de façon à maximiser ses profits totaux. On obtient immédiatement les conditions du premier ordre :

$$
\begin{aligned}
& \frac{d \Pi^{G}}{d m}=(1-t) \frac{d \pi_{1}^{A}}{d m}+(1-T) \frac{d \pi_{1}^{B}}{d m}=0 \\
& \frac{d \Pi^{G}}{d m^{*}}=(1-t) \frac{d \pi_{1}^{A}}{d m^{*}}+(1-T) \frac{d \pi_{1}^{B}}{d m^{*}}=0
\end{aligned}
$$

Les conditions (17a) et (17b) permettent de déterminer implicitement le

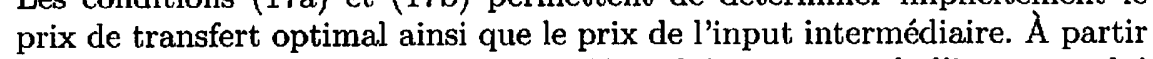
du moment où l'entreprise étrangère dépend étroitement de l'input que lui 
procure la multinationale, celle-ci peut décider de l'exclure du marché du bien final en augmentant très fortement le prix de l'input intermédiaire.

Proposition 5 Quand $t \geqslant T$ (y compris $t=T=0$ ) et que les revenus de source étrangère font l'objet d'un crédit d'impôt complet, alors il y a forclusion.

La raison en est relativement simple. Dans ce cas, la multinationale maximise ses profits sans tenir compte de la fiscalité dans le pays de résidence et dans le pays étranger. En utilisant le fait que que $\frac{\partial Q_{1}}{\partial m^{*}}>0$ et $\frac{\partial Q_{2}}{\partial m^{*}}<0$, la multinationale peut choisir un prix de l'input $m^{*}$ suffisamment élevé pour que $Q_{2}\left(m, m^{*}\right)=0$. Reste que si la multinationale est en situation de monopole sur le marché du bien final, elle est aussi en situation de monopole sur le marché du bien intermédiaire. Par conséquent, contraindre l'entreprise rivale à se retirer du marché expose la multinationale à des pertes de profits sur le bien intermédiaire. Elle doit donc faire un arbitrage. Cependant comme le montre la littérature sur les relations verticales (voir par exemple Tirole, 1988), quand un input fait l'objet d'un pouvoir de monopole et que son prix est supérieur au coût marginal, alors l'intégration verticale entre le monopoleur et les utilisateurs en amont permet de supprimer les markups successifs, ce qui accroît les profits de l'entreprise en question. Cela implique dans le cas de ce modèle que la forclusion augmente strictement le profit global de la multinationale. Evidemment si $t<T$ (ou s'il n'y a qu'un crédit d'impôt partiel sur les revenus de source étrangère), la forclusion n'est plus forcément souhaitable car le profit marginal retiré de la vente du bien final est plus faible et l'arbitrage entre le bien final et l'input intermédiaire se fait en faveur de celui-ci.

\section{Concurrence à la Bertrand et prix de transfert optimaux}

La littérature sur la politique commerciale stratégique montre généralement que la concurrence en prix inverse un certain nombre de résultats obtenus sous l'hypothèse de concurrence à la Cournot (Eaton et Grossman (1986)). Il apparaît en particulier dans les modèles où il $y$ a concurrence en prix sur un marché tiers que la politique optimale des gouvernements consiste à taxer les exportations plutôt qu'à les subventionner comme cela est le cas dans une situation de concurrence à la Cournot. En effet, une taxe sur les exportations a valeur d'engagement pour l'entreprise nationale car celleci peut choisir un prix plus élevé pour n'importe quel niveaux de prix de sa rivale. Les prix étant généralement considérés comme des compléments stratégiques, la taxe à l'exportation incite l'entreprise nationale à être moins agressive : le gouvernement induit en quelque sorte la firme concurrente à choisir un prix plus élevé, ce qui bénéficie à l'entreprise nationale. 
L'objet de cette section est de considérer l'impact d'une concurrence en prix sur la détermination du prix de transfert optimal. Sous l'hypothèse que $t<T$, le profit net global de la multinationale, $\Pi^{G}$, s'écrit :

$$
\begin{aligned}
\Pi^{G}= & (1-t) \pi_{1}^{A}+(1-T) \pi_{1}^{B} \\
= & (1-t)\left[r_{1}\left(p_{1}, p_{-1}\right)-c\left(q_{1}\left(p_{1}, p_{-1}\right)+Q_{1}\left(P_{1}, P_{-1}\right)\right)+m Q_{1}\left(P_{1}, P_{-1}\right)\right] \\
& +(1-T)\left[R_{1}\left(P_{1}, P_{-1}\right)-m(1+\tau) Q_{1}\left(P_{1}, P_{1}\right)\right]
\end{aligned}
$$

Nous supposons que la multinationale choisit à la première étape le prix de transfert qui lui permet de maximiser son profit net global, les deux filiales choisissant ensuite de façon décentralisées les prix. Nous mettrons l'accent sur l'effet stratégique sur le marché étranger en supposant que les coûts marginaux de production de la filiale exportatrice sont constants. La résolution de ce jeu se fait par récurrence amont de façon analogue au jeu précédent, ce qui nous permet d'obtenir directement la proposition suivante.

Proposition 6 Quand les entreprises se livrent à une concurrence à la Bertrand sur le seul marché étranger et que les deux filiales déterminent leurs décisions de production de façon décentralisée, le signe de $\left(m-\partial c / \partial Q_{1}\right)$ dépend de deux effets qui jouent en sens opposé :

(i) un effet " transfert de bénéfice »: $\left[\frac{(\tau-\phi) Q_{1}}{d Q_{1} / d m}\right]<0(>0)$

$$
\text { si } \tau>\phi(\tau<\phi) \text {. }
$$

(ii) un effet " stratégique ":

$$
-(N-1) \cdot\left(d Q_{1} / d m\right)^{-1} \cdot\left[\frac{\partial R_{1}}{\partial P_{l}}-m(1+\tau) \cdot \frac{\partial Q_{1}}{\partial P_{l}}\right] \cdot \frac{\partial P_{l}}{\partial m}>0
$$

Preuve - Annexe A.1.

La proposition 6 permet de retrouver l'effet stratégique mis en exergue précédemment mais son signe n'est plus le même quand il y a concurrence à la Bertrand sur le marché étranger. En effet, l'effet stratégique sur le marché étranger plaide désormais pour un prix de transfert supérieur au coût marginal. L'intuition est la suivante : toutes choses égales par ailleurs, un prix de transfert élevé conduit la filiale située dans le pays étranger (pays $\mathrm{B}$ ) à augmenter son prix de vente. Mais alors la meilleure réponse des entreprises concurrentes (qui sont supposées observer parfaitement le prix de transfert fixé par la multinationale) consiste à adopter elles aussi un prix de vente élevé, ce qui est évidemment favorable à la filiale du pays $B$ en terme de profit (on suppose à la suite de Eaton et Grossman (1986) que les prix sont des compléments stratégiques et par conséquent que les pentes des fonctions de réaction ont une pente positive).

C'est précisément sur ce point que s'explique la différence de résultats entre la concurrence à la Cournot et la concurrence à la Bertrand. Dans le premier cas, quand la filiale a une stratégie agressive en augmentant ses quantités vendues, ses concurrentes réagissent en se repliant (c'est à dire 
en vendant moins). Dans le second cas, si la filiale baissait, son prix de vente, cela conduiraient ses concurrentes à réagir de façon plus agressive en baissant elles aussi leur prix de vente.

Proposition 7 Dans le cas où la filiale exportatrice située dans le pays A est en situation de monopole sur son marché $(M=1)$ et que les taux d'impôt et le droit de douane sont nuls $(t=T=\tau=0)$, alors il est toujours vrai que $m>\partial c / \partial Q_{1}$.

En revanche, dès lors que les taux d'impôt et le droit de douane ne sont plus nuls $(t>0 ; T>0 ; \tau>0)$, l'effet « transfert de bénéfice » réapparaît et son signe dépend comme précédemment de $(\tau-\phi)$. La combinaison de ces deux effets ne permet pas de conclure à une sur-évaluation ou au contraire à une sous-évaluation du prix de transfert. Il apparaît cependant comme on pouvait s'y attendre intuitivement que la plupart des résultats sont inversés par rapport à la concurrence à la Cournot.

\section{Conclusion}

L'objet de cet article était de montrer que lorsque les décisions de production ou de prix sont décentralisées au niveau des filiales et que celles-ci sont en concurrence avec d'autres entreprises, alors le prix de transfert optimal n'est plus égal au coût marginal de production de la filiale exportatrice, même quand l'on fait abstraction des taux d'imposition et des droits de douane. Il apparaît plus particulièrement que la nature de la concurrence est importante, notamment celle qui prévaut sur le marché étranger. Dans le cas d'une concurrence à la Cournot dans le pays $\mathrm{B}$, la multinationale a intérêt à sous-évaluer son prix de transfert de façon à permettre à sa filiale d'être plus agressive par rapport à ses rivales en augmentant les quantités vendues. En revanche, dans le cas d'une concurrence à la Bertrand, sous-évaluer le prix de transfert conduirait les entreprises rivales à baisser leur prix de vente et à être plus agressives. Nous montrons cependant que l'« effet stratégique ", quelle que soit la nature de la concurrence, est contrecarré par un effet rétroactif sur la marché où opère la filiale exportatrice (marché national). Par exemple, dans le cas d'une concurrence à la Cournot, l'augmentation des quantités vendues à la filiale située à l'étranger se traduit par une augmentation du coût marginal de production de la filiale exportatrice. Cette dernière réduit donc ses quantités vendues (au profit de ses rivales) et son niveau de profit diminue. Toutes choses égales par ailleurs, l'effet rétroactif réduit l'incitation de la multinationale à sous-estimer son prix de transfert (et inversement dans le cas d'une concurrence en prix). L'hypothèse sousjacente à ces résultats est que l'ensemble des partie (y compris les entreprises concurrentes des deux filiales de la multinationale) obervent parfaitement le prix de transfert fixé par la multinationale. Une extension du modèle serait de considérer que le prix de transfert est une information privée des 
entreprises affiliées et que celui-ci peut être sur-évalué (par rapport au coût marginal) ou sous évalué. Cela modifierait bien évidemment la réponse des entreprises concurrentes.

\section{Bibliographie}

Bond, E.W. (1980), "Optimal Transfer Pricing when Tax Rates Differ", Southern Economic Journal, 47, pp.191-200.

Brander, J. et B.J. Spencer (1985), "Export Subsidies and International Market Share Rivalry", Journal of International Economics, 18, pp.488-511.

Brander, J. (1995), "Strategic Trade Policy"; in Gene Grossman et Ken Rogoff (eds), The handbook of Interntional Economics, vol. N³, NorthHolland.

Bulow, J.I., J.D. Geanakopulos et P.D. Klemperer (1985), "Multimarket Oligopoly : Strategic Substitutes and Complements", Journal of Political Economy, 93, 3,pp. 488-511.

Copithorne, L. W. (1971), "International Corporate Transfer Prices and Government Policy", Canadian Journal of Economics, 4, pp.324-341.

Delage, B. (1999), «Concurrence fiscale : un survol », L'actualité économique, 75, pp.67-92.

Eaton, J. et G.M. Grossman (1986), "Optimal Trade and Industrial Policy under Oligopoly", Quaterly Journal of Economics, pp.383-406.

Eden, L. (1978), "Vertically Integrated Multinationals : a Microeconomic Analysis", The canadian Journal of Economics, 11, pp.534-546.

Hines, J.R. (1996), "Tax Policy and The Activities of Multinational Corporations", working paper n 5589 , National Bureau of Economic Research.

Hirshleifer, J. (1956), "On The Economics of Transfer Pricing", Journal of Business, 29, pp.172-184.

Horst, T. (1971), "The Theory of the MNE : Optimal Behavior Under Different Tariff and Tax Rates", Journal of Political Economy, 79, pp.10591072.

Hugounenq, R., J. Le Cacheux et Th. Madiès (1999), « Risques de concurrence fiscale en Europe ", Revue de l'OFCE, $\mathrm{n}^{\circ} 70, \mathrm{pp} .63-109$.

Janeba, E. (1997), International Tax Competition, Mohr Siebeck, Tubingen.

Kant, C. (1990), "Multinational Firms and Government Revenues", Journal of Public Economics, 42, pp.135-147.

Lall, S. (1978), "The Pattern of Intra-firm exports by U.S. Multinationals, Oxford Bulletin of Economics and Statistics, 40, pp.209-222. 
OCDE (1995), Principes applicables en matière de prix de transfert à l'intention des entreprises multinationales et des administrations fiscales, Paris.

Schjelderup, G. et L. Sorgard (1997), "Transfer Pricing as a Strategic Device for Decentralized Multinationals", International Tax and Public Finance, 4, pp.277-290.

\section{Annexe A.1 Preuve de la proposition 6}

L'effet d'une modification à la marge de $m$ sur le profit global de la multinationale s'écrit de la façon suivante :

$$
\begin{aligned}
\frac{d \Pi^{G}}{d m}= & (1-t)\left[\left(\frac{\partial r_{1}}{\partial p_{1}}-\frac{\partial c}{\partial q_{1}} \cdot \frac{\partial q_{1}}{\partial p_{1}}\right) \cdot \frac{\partial p_{1}}{\partial Q_{1}} \cdot \frac{d Q_{1}}{d m}\right. \\
& +\sum_{j \neq 1}^{M}\left(\frac{\partial r_{1}}{\partial p_{j}}-\frac{\partial c}{\partial q_{1}} \cdot \frac{\partial q_{1}}{\partial p_{j}}\right) \cdot \frac{\partial p_{j}}{\partial p_{1}} \cdot \frac{\partial p_{1}}{\partial Q_{1}} \cdot \frac{d Q_{1}}{d m} \\
& \left.-\frac{\partial c}{\partial Q_{1}} \cdot \frac{d Q_{1}}{d m}+Q_{1}+m \frac{d Q_{1}}{d m}\right] \\
& +(1-T)\left[\left(\frac{\partial R_{1}}{\partial P_{1}}-m(1+\tau) \cdot \frac{\partial Q_{1}}{\partial P_{1}}\right) \cdot \frac{\partial p_{1}}{\partial m}\right. \\
& \left.+\sum_{l \neq 1}^{N}\left(\frac{\partial R_{1}}{\partial P_{j}}-m(1+\tau) \cdot \frac{\partial Q_{1}}{\partial P_{j}}\right) \cdot \frac{\partial P_{j}}{\partial m}-(1+\tau) Q_{1}\right]
\end{aligned}
$$

où $\frac{d Q_{1}}{d m}=\frac{\partial Q_{1}}{\partial P_{1}} \cdot \frac{\partial P_{1}}{\partial m}+\sum_{l \neq 1}^{N} \frac{\partial Q_{1}}{\partial P_{l}} \cdot \frac{\partial P_{l}}{\partial m}$.

Les deux filiales maximisant leur profit et décidant des prix de vente de leur produit, les conditions du premier ordre sont:

$$
\begin{aligned}
& \frac{\partial \pi_{1}^{A}}{\partial p_{1}}=(1-t)\left[\frac{\partial r_{1}}{\partial p_{1}}-\frac{\partial c}{\partial q_{1}} \cdot \frac{\partial q_{1}}{\partial p_{1}}\right]=0 \\
& \frac{\partial \pi_{2}^{B}}{\partial P_{1}}=(1-T)\left[\frac{\partial R_{1}}{\partial P_{1}}-(1+\tau) m \cdot \frac{\partial Q_{1}}{\partial P_{1}}\right]=0
\end{aligned}
$$

En substituant (A.2) et (A.3) dans (A.1) et en réarrangeant les termes, on obtient :

$$
m-\frac{\partial c}{\partial Q_{1}}=\frac{(1-T)}{(1-t)} \cdot\left[\frac{d Q_{1}}{d m}\right]^{-1} \cdot\left[(\tau-\phi) Q_{1}\right.
$$




$$
\begin{aligned}
& -(N-1) \cdot\left(\frac{\partial R_{1}}{\partial P_{l}}-m(1+\tau) \cdot \frac{\partial Q_{1}}{\partial P_{l}}\right) \cdot \frac{\partial P_{l}}{\partial m} \\
& \left.-(M-1) \cdot \frac{(1-t)}{(1-T)}\left(\frac{\partial r_{1}}{\partial p_{j}}-\frac{\partial c}{\partial q_{1}} \cdot \frac{\partial q_{1}}{\partial p_{j}}\right) \cdot \frac{\partial p_{j}}{\partial p_{1}} \cdot \frac{\partial p_{1}}{\partial Q_{1}}\right]
\end{aligned}
$$

En posant $M=1$ ou en supposant que le coût marginal de production de la filiale exportatrice est constant, L'équation (A.4) permet de retrouver directement les deux effets qui jouent sur la détermination du prix de transfert optimal. Il suffit ensuite de signer chacun de ces effets. La preuve de (i) est évidente. (ii) est positif car (1) $\frac{\partial \pi_{2}}{\partial P_{t}} \equiv\left[\frac{\partial R_{1}}{\partial P_{t}}-m(1+\tau) \cdot \frac{\partial Q_{1}}{\partial P_{t}}\right]>0$ (les prix sont des compléments stratégiques et par conséquent les pentes des fonctions de réaction sont de pente positive (voir sur ce point Janeba (1996)); (2) $\frac{\partial P_{1}}{\partial m}=\frac{\partial P_{l}}{\partial P_{1}} \cdot \frac{\partial P_{1}}{\partial m}>0$ car la concurrence à la Bertrand implique que pour un grand nombre de fonctions de demande et de coût, $\frac{\partial P_{l}}{\partial P 1}>0$ (voir Burlow et alii (1985)); (3) $\frac{d Q_{1}}{d m}=\frac{\partial Q_{1}}{d P_{1}} \cdot \frac{\partial P_{1}}{\partial m}+(N-1) \frac{\partial Q_{1}}{\partial P_{1}} \cdot \frac{\partial P_{1}}{\partial P_{1}} \cdot \frac{\partial P_{1}}{\partial m}<0$. 